\title{
Fermented Astragalus in diet improved laying performance, egg quality, antioxidant and immunological status and intestinal microbiota in laying hens
}

\author{
Hong-Tao Shi ${ }^{1,2+}$, Bai-Yu Wang ${ }^{3 \dagger}$, Chuan-Zhou Bian ${ }^{1}$, Ying-Qian Han ${ }^{3 *}$ and Hong-Xing Qiao ${ }^{1^{*}}$
}

\begin{abstract}
In the era of increased antibiotic resistance and ever-stricter control on antibiotic use, it is urgent to develop green, safe, and non-residue alternatives to antibiotics applied to the poultry industry. To this end, we supplied the potential Lactobacillus plantarum (L. plantarum) fermented Astragalus in the diet of laying hens, with a final addition of 3\%o. Its effects have been assessed on laying performance, egg quality, antioxidant and immunological status, and intestinal microbiota, and are compared to the control group, to the Astragalus group containing 3\%o unfermented Astragalus, and to the L. plantarum group containing $2 \%$ L. plantarum $\left[5 \times 10^{8}\right.$ colony-forming unit (CFU) per milliliter (mL)]. During the second half of the experimental period (15 to 28 days), the egg production rate was considerably higher in the fermented Astragalus group than that in the other groups, with the fermented Astragalus group having the lowest feed conversion ratio. No significant difference $(P>0.05)$ was noted among treatments on egg quality. Fermented Astragalus-treated hens exhibited significantly increased catalase (CAT), glutathione peroxidase (GSH-Px), superoxide dismutase (SOD) and total antioxidant capacity (T-AOC) in serum, and reduced malondialdehyde (MDA) in serum. Furthermore, fermented Astragalus supplementation resulted in a significant increase in ileal microbiota abundance relative to control. In conclusion, feeding laying hens with L. plantarum fermented Astragalus has beneficial effects on production, antioxidant potential, immunity, and ileal microbiota. L. plantarum fermented Astragalus is expected to be a novel feed additive used in poultry production.
\end{abstract}

Keywords: Fermented astragalus, Laying hens, Egg quality, Antioxidant and immunological status, 16S rRNA, Intestinal microbiota

\section{Introduction}

Eggs are one of the most crucial sources of animal protein and nutritional content in human diets. Due to the widespread use of antibiotics in poultry, drug residues in eggs have been gaining worldwide concern over the

\footnotetext{
*Correspondence: twgjl@163.com; zzmzqhx@163.com

${ }^{\dagger}$ Hong-Tao Shi and Bai-Yu Wang contributed equally to this work

${ }^{1}$ College of Veterinary Medicine, Henan University of Animal Husbandry and Economy, Zhengzhou 450046, Henan, China

${ }^{3}$ College of Animal Sciences and Veterinary Medicine, Henan Agricultural University, Zhengzhou 450046, Henan, China

Full list of author information is available at the end of the article
}

past few years (Vandemaele et al. 2002). In addition, antibiotic abuse has led to intestinal dysbacteriosis, diarrhea, immunocompromised state (Willing et al. 2011). Thus, it is imperative to develop green, safe, and non-residue alternatives to antibiotics applied to the poultry industry. Traditional Chinese herbal medicines are the gem of China with characteristics of safety, efficiency, and low residue and are commonly used in preventive or therapeutic strategies for animal diseases (Xu et al. 2017). Traditional Chinese herbal medicines have utilised as feed additives for growth promotion and improvement of immunity and various effects, 
including anti-bacterial, anti-viral and antioxidative activities (Patra et al. 2015; Wang et al. 2015). Since ancient times, traditional Chinese herbal medicines can be processed by microbial fermentation for improving its quality (Zhu et al. 2014). For example, fermentation of Chinese herbal medicine mediated by microbes can degrade macromolecule-materials into small ones and reduce their side effects (Ai et al. 2019). Because microorganisms and their metabolic products can regulate the bioactive products of traditional Chinese herbal medicines, there is a close relationship between microorganisms and traditional Chinese herbal medicines.

Astragalus is a universal traditional Chinese herbal medicine and its main active pharmaceutical ingredients include polysaccharides, saponins, flavonoids, anthraquinones, alkaloids, amino acids, $\beta$-sitosterol and metallic elements (Li et al. 2014). Astragalus has been reported to possess anti-inflammatory (Kim et al. 2013), anti-viral (Kallon et al. 2013) and antioxidant (Shahzad et al. 2016) activities and to enhance immunity (Qin et al. 2012), and it has been widely used in livestock. Nevertheless, challenges to the extraction yield of Astragalus functional ingredients are raised due to the recalcitrance of plant cell walls, and novel strategies for the improvement of Astragalus utilization efficiency have to be focused. The trend of microbial fermentation offers the possibility of addressing the above problem. In recent years, research revealed that utilizing the fungus Aspergillus to ferment the Astragalus can significantly increase its phenolic contents and antioxidant activity, and the solid-state bioprocessing strategy could be an innovative approach to enhance the antioxidant activity of Astragalus (Sheih et al. 2011). Our previous studies have verified that the solid fermentation of Astragalus by L. plantarum promotes the extraction yield of Astragalus active components and the production yield of organic acids (Qiao et al. 2018c). Further investigation showed that fermented Astragalus improves broiler growth performance, enhances serum antioxidant status, and reduces fecal pathogenic microbiota of broiler chickens (Qiao et al. 2018b).

Over the last few years, there has meant considerable research on the application of Astragalus polysaccharide as a feed additive in livestock including laying hens. However, there has not been a systematic appraisal of the application of Astragalus fermented by L. plantarum as a feed additive for laying hens. In this study, we investigated the possible effects of Astragalus fermented by $L$. plantarum on egg production, egg quality, antioxidant status, immune factors expression and gut microbiome of laying hens, combining the classical culture and detection methods with high throughput sequencing.

\section{Materials and methods}

Fermentation of Astragalus

Lactobacillus plantarum (CGMCC 1.557) was purchased from the China General Microbiological Culture Collection Center (CGMCC) (Beijing, China). Te dried root of Astragalus membranaceus (Fisch.) Bge. var. mongholicus was obtained from Gansu Huisen Pharmaceutical Development Co., Ltd. (Minxian, Gansu, China) and verified by Dr. JingYu Zhang (Henan University of Traditional Chinese Medicine, Zhengzhou, Henan, China). The purchased Astragalus was crushed into powder and filtered with a 100-mesh filter for further studies. The fermentation of Astragalus was performed following the method reported in our previous publications with slight modification (Qiao et al. 2018b). Briefly, dried Astragalus powder (7500 g) was inoculated with $L$. plantarum $\left(1 \times 10^{6} \mathrm{CFU}\right.$ per gram $)$ with a water content of $45 \%$ and Astragalus-L. plantarum mixtures were divided equally into $35 \times 45-\mathrm{mm}$ plastic film bags. The bags were sealed for fermentation at $37{ }^{\circ} \mathrm{C}$ for 30 days and then dried out at room temperature for future use.

\section{Experimental design, diets and management}

Two hundred and forty healthy Hy-Line Gray hens (351 days, Zhengzhou, China) were acclimated with the basal diets for 7 days. Then, hens were randomly divided into four groups (fermented Astragalus group, Astragalus group, L. plantarum group, and control group), each containing five replicates, with 12 hens per replicate. The control group was fed with the basal diet; the L. plantarum group was fed with the basal diet supplemented with $2 \%$ Lactobacillus solution $\left(5 \times 10^{8} \mathrm{CFU} /\right.$ $\mathrm{mL}$ ) through uniform spraying; the Astragalus group was fed with the basal diet supplemented with 3\%o Astragalus, and fermented Astragalus group was fed with the basal diet supplemented with 3\%o fermented Astragalus (pre-experimental results showed that supplementing at a rate of $3 \%$ of diet achieves optimal results). The trial lasted for 35 days (7-day adaptation period and 28-day experimental stage). The hens were housed in a clean environment with good ventilation and artificial lighting allowed $16 \mathrm{~h}$ of lighting per day, and with water and food ad libitum. The basal diet of all groups was the same and prepared according to the NRC (1994) laying hen nutrition requirement standard. The composition and nutrient levels of the basal diet were shown in Table 1 . All animal experiments were conducted according to the Guidelines for the Care and Use of Experimental Animals established and approved by the Laboratory Animal Management Committee of Henan University of Animal Husbandry and Economy (HNMY 1606). 
Table 1 Composition and nutrient levels of the basal diet (air-dry basis \%)

\begin{tabular}{lclc}
\hline Ingredients & Content & Nutrient levels & Content \\
\hline Corn & 61.4 & $\mathrm{ME} /(\mathrm{MJ} / \mathrm{kg})^{\mathrm{b}}$ & 11.01 \\
Soybean meal & 23.8 & $\mathrm{CP}$ & 15.49 \\
Wheat bran & 2.0 & $\mathrm{Ca}$ & 3.50 \\
Soybean oil & 0.6 & $\mathrm{TP}$ & 0.56 \\
CaHPO $_{4}$ & 1.3 & $\mathrm{AP}$ & 0.35 \\
Limestone $_{\text {NaCl }}$ & 8.6 & $\mathrm{Lys}$ & 0.74 \\
Premix & 0.3 & Met + Cys & 0.51 \\
Total & 2.0 & & \\
\hline
\end{tabular}

a The premix provided the following per kilogram of the diet: $\mathrm{VA} 11,000 \mathrm{IU}, \mathrm{VD}_{3}$ $3200 \mathrm{IU}, \mathrm{VE} 25 \mathrm{IU}, \mathrm{VK}_{3} 2.2 \mathrm{mg}, \mathrm{VB}_{1} 1.5 \mathrm{mg}, \mathrm{VB}_{2} 3.5 \mathrm{mg}, \mathrm{VB}_{12} 3 \mathrm{mg}$, nicotinic $28 \mathrm{mg}$, calcium pantothenate $8.5 \mathrm{mg}$, biotin $0.5 \mathrm{mg}$, choline $255 \mathrm{mg}$, Fe $55 \mathrm{mg}, \mathrm{Zn}$ $62 \mathrm{mg}$, Cu $6 \mathrm{mg}$, Se $0.20 \mathrm{mg}$

b ME was a calculated value, while the others were measured values

\section{Hen productivity and egg quality}

During the experimental period, egg production, broken egg production, egg weight, and feed intake were recorded daily. The egg production rate and the feed conversion ratio (FCR) (feed intake/egg weight gain) during day 1 to day 14 and day 15 to day 28 were calculated to assess the laying performance. On day 14 and day 28 , five eggs from each replicate were randomly sampled and measured egg quality parameters of egg shape index (ESI), eggshell strength (ESS), eggshell thickness (EST), albumen height $(\mathrm{AH})$, Haugh unit $(\mathrm{HU})$, yolk color $(\mathrm{YC})$ and yolk weight ( $\mathrm{YW})$.

\section{Serum antioxidant indices}

On day 14 and day 28, one hen from each replicate was randomly selected. Following blood collection from the heart, the serum was isolated and stored at $-20{ }^{\circ} \mathrm{C}$ until use. The CAT assay kit (Catalog number R22072), GSH-Px assay kit (Catalog number R21876), SOD assay kit (Catalog number R22262), T-AOC assay kit (Catalog number R24147) and MDA assay kit (Catalog number R21869) were purchased from Shanghai yuanye BioTechnology Co., Ltd (Shanghai, China).

\section{Real-time qPCR}

After blood samples collection, liver, spleen, ileum, and cecum samples were harvested for interferon-gamma $(\mathrm{IFN}-\gamma)$ and tumor necrosis factor-alpha (TNF- $\alpha$ ) mRNA expression evaluation. Total RNA was extracted from these tissues using RNAiso Plus (Catalog number 9108, Takara, Otsu, Shiga, Japan) and reverse transcribed into cDNA with PrimeScript ${ }^{\mathrm{TM}} \mathrm{RT}$ reagent Kit with gDNA Eraser (Perfect Real Time) (Catalog number
Table 2 Primers used for quantitative real-time PCR analysis

\begin{tabular}{lll}
\hline Primers & Sequences $\mathbf{5}^{\prime} \sim \mathbf{3}^{\prime}$ & Size \\
\hline IFN- $\gamma-F$ & AACAACCTTCCTGATGGCGT & $107 \mathrm{bp}$ \\
IFN- - - & TGAAGAGTTCATTCGCGGCT & \\
$\beta-a c t i n-F$ & TATGTGCAAGGCCGGTTTCG & $170 \mathrm{bp}$ \\
$\beta-a c t i n-R$ & CAATGGGGTACTTCAGGGTCAG & \\
TNF- $-\mathrm{F}$ & GCCCTTCCTGTAACCAGATG & $71 \mathrm{bp}$ \\
TNF- $a-R$ & ACACGACAGCCAAGTCAACG & \\
\hline
\end{tabular}

RR047, Takara, Otsu, Shiga, Japan) according to the manufacturer's protocol. The primers used in the study were synthesized by Sangon Biotech (Shanghai) Co., Ltd. (Shanghai, China), and primer sequences are summarized in Table 2. The Real-time qPCR reactions were performed using a TB Green Premix EX Taq (Catalog number RR420, Takara, Otsu, Shiga, Japan) in a 7500 Fast Real-Time PCR System (Thermo Fisher). $\beta$-actin was used as a housekeeping gene. The relative mRNA expression levels of the target genes compared to the housekeeping gene were calculated using the $2^{-\Delta \Delta \mathrm{Ct}}$ method.

\section{Sample collection and DNA extraction}

On day 14 and day 28 , a total of 48 hens were randomly selected (12 hens per group) and euthanized via an intravenous injection of pentobarbital sodium $(150 \mathrm{mg} / \mathrm{kg})$ to collect ileal and cecal contents (Pan et al. 2018). The samples were named as the 14-d ileum control group (14IA), 14-d ileum Astragalus group (14IB), 14-d ileum L. plantarum group (14IC), 14-d ileum fermented Astragalus group (14ID), 14-d cecum control group (14CA), 14-d cecum Astragalus group (14CB), 14-d cecum L. plantarum group (14CC), 14-d cecum fermented Astragalus group (14CD), 28-d ileum control group (28IA), 28-d ileum Astragalus group (28IB), 28-d ileum L. plantarum group (28IC), 28-d ileum fermented Astragalus group (28ID), 28-d cecum control group (28CA), 28-d cecum Astragalus group (28CB), 28-d cecum L. plantarum group (28CC), and 28-d cecum fermented Astragalus group (28CD). All collected samples were immediately stored at $-20{ }^{\circ} \mathrm{C}$ until extraction. DNA extraction was performed with a commercial DNA extraction kit (Tiangen Biotech Corporation, Beijing, China) and quantified by a Qubit 2.0 fluorometer (Invitrogen Corporation, Carlsbad, CA, USA). The extracted DNA was qualitatively assessed by $0.8 \%$ agarose gel electrophoresis and spectrophotometry (optical density at $260 / 280 \mathrm{~nm}$ ) and stored at $-20^{\circ} \mathrm{C}$ until further analysis. 


\section{S rRNA gene sequencing and analysis}

For amplicon library generation, the V4 region of the $16 \mathrm{~S}$ rRNA gene of all DNA samples was amplified with gene-specific primers (F: 5' - AYTGGGYDTAAAGNG-3'; R: 5'-TACNVGGGTATCTAATCC-3'). PCR amplifications were performed using Q5 high-fidelity PCR DNA polymerase (Catalog number M0491, New England Biolabs, Ipswich, MA, USA) and completed under the following conditions: a pre-denaturation at $98{ }^{\circ} \mathrm{C}$ for $30 \mathrm{~s}$; 27 cycles of $98{ }^{\circ} \mathrm{C}$ for $15 \mathrm{~s}, 50{ }^{\circ} \mathrm{C}$ for $30 \mathrm{~s}$, and $72{ }^{\circ} \mathrm{C}$ for $30 \mathrm{~s}$; a final extension at $72{ }^{\circ} \mathrm{C}$ for $5 \mathrm{~min}$. Amplicons were purified using the Axygen AxyPrep DNA Gel Extraction Kit (Catalog number AP-GX-250G, Corning Life Sciences, Corning, NY, USA). DNA libraries were validated and quantified using the TruSeq Nano DNA LT Library Preparation Kit (Catalog number FC-121-4001, Illumina, San Diego, CA, USA) and Quant-iT ${ }^{\mathrm{TM}}$ PicoGreen $^{\mathrm{TM}}$ dsDNA Assay Kit (Catalog number P11496, Invitrogen Corporation, Carlsbad, CA, USA). After quantification, the barcoded $\mathrm{V} 4$ amplicons were pooled to a final concentration of $2 \mathrm{nmol} / \mathrm{L}$ and sequenced using an Illumina MiSeq platform to generate $300 \mathrm{bp}$ paired-end reads. Raw reads were quality-filtered to remove any reads less than 150 bp using Quantitative Insights into Microbial Ecology (QIIME) version 1.8 (Caporaso et al. 2010) and clustered into Operational Taxonomic Units (OTUs) based on a $97 \%$ similarity threshold. The representative sequence was chosen based on the abundance and was aligned under a given taxonomic classification using the Greengenes database, and low abundance OTUs of archaea and eukaryotes were removed (Bokulich et al. 2013). Alpha-diversity was calculated with Chao1 and ACE estimators, Shannon and Simpson indices. Partial least squares discriminant analysis (PLS-DA) was performed using QIIME software package v1.8 to discriminate between different groups (day 14 and day 28) and to establish $\beta$-diversity. The sequences generated in this study have been deposited in the National Center for Biotechnology Information sequence read archive (https:// www.ncbi.nlm.nih.gov/biosample) under the Accession number SRA: PRJNA533918.

\section{Statistical analysis}

Only for genes mRNA expression assay, statistical analyses were performed with Student's t-test and graphed using GraphPad Prism 6.00 (GraphPad Software), and significance levels are indicated as ${ }^{*} P<0.05,{ }^{* * *} P<0.01$, ${ }^{* * * * *} P<0.001,{ }^{* * * * *} P<0.0001$. All other statistical analyses were performed by one-way analysis of variance using SPSS 24.0 software, and all data were expressed as means $\pm \mathrm{SD}$, with $P<0.05$ considered statistically significant.

\section{Results}

Hen productivity and egg quality

The effects of different dietary supplements on the laying hen production performance and egg quality are listed in Tables 3 and 4. During day 1 to day 14, there were no differences in the laying rate and FCR among four groups $(P>0.05)$, with hens fed with fermented Astragalus had the highest laying rate. During day 15 to day 28, hens fed with fermented Astragalus had the highest laying rate, $7.14 \%$ higher than that of the control group $(P<0.05)$. Although the differences were not statistically significant $(P>0.05)$, the laying rate of the Astragalus group and L. plantarum group were also increased by $3.25 \%$ and $2.99 \%$, respectively in comparison with the control group. Furthermore, the FCR of the fermented Astragalus group was reduced by $6.6 \%$ compared with that of the control group $(P<0.05)$, while the FCR of the Astragalus group and L. plantarum group displayed no significant differences as compared with the controls $(P>0.05)$. In addition, no significant differences in the phenotype of the egg quality including ESI, ESS, EST, AH, YC, HU, and YW were observed among dietary treatments, suggesting that dietary supplements have no significant effects on egg quality in this study. Therefore, we identified that dietary supplementation of fermented Astragalus can

Table 3 Effects of different dietary supplements on the production performance of laying hens

\begin{tabular}{|c|c|c|c|c|c|c|}
\hline Parameters & Control & Astragalus & L.plantarum & Fermented Astragalus & $P$-value & SEM \\
\hline \multicolumn{7}{|l|}{$1-14$ days } \\
\hline Laying rate, \% & $87.50 \pm 3.45$ & $89.17 \pm 2.08$ & $87.50 \pm 2.70$ & $90.60 \pm 3.80$ & 0.349 & 1.376 \\
\hline FCR & $2.02 \pm 0.08$ & $1.94 \pm 0.05$ & $2.02 \pm 0.10$ & $1.95 \pm 0.08$ & 0.224 & 0.037 \\
\hline \multicolumn{7}{|l|}{$15-28$ days } \\
\hline Laying rate, \% & $88.96 \pm 3.76 a$ & $92.21 \pm 2.75 a b$ & $91.95 \pm 3.83 a b$ & $96.10 \pm 1.03 b$ & 0.017 & 1.368 \\
\hline FCR & $1.81 \pm 0.08 b$ & $1.75 \pm 0.06 a b$ & $1.77 \pm 0.11 a b$ & $1.69 \pm 0.05 a$ & 0.172 & 0.035 \\
\hline
\end{tabular}

Different lowercase letters in the same row indicate significant difference $(P<0.05)$, and the same letters or no letters indicate no significant difference $(P>0.05)$ (same as below)

FCR feed conversion ratio 
Table 4 Effects of different dietary supplements on the egg quality

\begin{tabular}{|c|c|c|c|c|c|c|}
\hline Parameters & Control & Astragalus & L. plantarum & Fermented Astragalus & $P$-value & SEM \\
\hline \multicolumn{7}{|l|}{14 days } \\
\hline ESI & $1.29 \pm 0.02$ & $1.29 \pm 0.03$ & $1.29 \pm 0.03$ & $1.29 \pm 0.03$ & 0.961 & 0.012 \\
\hline $\mathrm{ESS}(\mathrm{kg} / \mathrm{N})$ & $3.59 \pm 0.41$ & $4.04 \pm 0.33$ & $3.87 \pm 0.32$ & $3.90 \pm 0.40$ & 0.302 & 0.164 \\
\hline $\mathrm{EST}(\mathrm{mm})$ & $0.344 \pm 0.013$ & $0.344 \pm 0.011$ & $0.346 \pm 0.008$ & $0.348 \pm 0.008$ & 0.927 & 0.005 \\
\hline $\mathrm{AH}(\mathrm{mm})$ & $8.74 \pm 0.43 a b$ & $8.74 \pm 0.63 a b$ & $8.47 \pm 0.51 a$ & $9.21 \pm 0.33 b$ & 0.162 & 0.219 \\
\hline YC & $5.15 \pm 0.32$ & $5.00 \pm 0.28$ & $5.44 \pm 0.21$ & $5.32 \pm 0.40$ & 0.160 & 0.139 \\
\hline $\mathrm{HU}$ & $92.84 \pm 1.90$ & $92.16 \pm 3.42$ & $92.10 \pm 2.82$ & $94.75 \pm 1.61$ & 0.183 & 1.138 \\
\hline YW & $15.75 \pm 0.37$ & $15.47 \pm 0.32$ & $15.60 \pm 0.22$ & $15.27 \pm 0.73$ & 0.417 & 0.202 \\
\hline \multicolumn{7}{|l|}{28 days } \\
\hline ESI & $1.28 \pm 0.02$ & $1.28 \pm 0.01$ & $1.29 \pm 0.01$ & $1.29 \pm 0.03$ & 0.452 & 0.008 \\
\hline ESS (kg/N) & $4.31 \pm 0.37$ & $4.34 \pm 0.60$ & $4.20 \pm 0.16$ & $4.54 \pm 0.41$ & 0.625 & 0.185 \\
\hline $\mathrm{EST}(\mathrm{mm})$ & $0.375 \pm 0.014$ & $0.392 \pm 0.020$ & $0.389 \pm 0.013$ & $0.387 \pm 0.018$ & 0.396 & 0.007 \\
\hline $\mathrm{AH}(\mathrm{mm})$ & $8.80 \pm 0.40$ & $8.92 \pm 0.43$ & $8.85 \pm 0.24$ & $9.08 \pm 0.44$ & 0.676 & 0.173 \\
\hline$Y C$ & $5.59 \pm 0.39$ & $5.55 \pm 0.47$ & $5.22 \pm 0.12$ & $5.44 \pm 0.45$ & 0.435 & 0.170 \\
\hline $\mathrm{HU}$ & $92.57 \pm 2.19$ & $92.80 \pm 2.42$ & $92.74 \pm 1.03$ & $93.80 \pm 2.07$ & 0.763 & 0.895 \\
\hline YW & $15.40 \pm 0.39$ & $15.50 \pm 0.59$ & $15.78 \pm 0.38$ & $15.76 \pm 0.58$ & 0.556 & 0.222 \\
\hline
\end{tabular}

ESl egg shape index, ESS eggshell strength, EST eggshell thickness, $A H$ albumen height, $H U$ haugh unit, $Y C$ yolk color, $Y W$ yolk weight

markedly improve egg production and decrease FCR, and the effect is substantially superior to that of Astragalus and L. plantarum.

\section{Serum antioxidant indices}

The effects of different dietary supplements on the laying hen antioxidant status are listed in Table 5. The data indicated that all dietary supplementation did not have an effect on the biomarkers of antioxidative stress at day $14(P>0.05)$. However, serum CAT, GSH-Px, SOD, and $\mathrm{T}$-AOC concentrations were increased by $61.5 \%, 62.4 \%$, $68.0 \%$, and $52.6 \%(P<0.05)$ at the end of experimentation in the fermented Astragalus group as compared with the controls. No statistically significant differences were observed for CAT, GSH-Px, and SOD among the control, Astragalus, and L. plantarum groups $(P>0.05)$. Among the effects of different dietary supplements on MDA activity in serum of laying hens, hens fed with fermented Astragalus, Astragalus diet were significantly decreased by $54.7 \%$ and $43.0 \%$ than that of the control treatment $(P<0.05)$; treatment with $L$. plantarum diet did not dramatically differ from the control treatment $(P>0.05)$. The results presented above show that dietary supplementation of fermented Astragalus can markedly improve laying hen antioxidant status, and the effect is significantly superior to that of Astragalus and L. plantarum.

Table 5 Effects of different dietary supplements on the antioxidant status of laying hens

\begin{tabular}{|c|c|c|c|c|c|c|}
\hline Parameters & Control & Astragalus & L. plantarum & Fermented Astragalus & $P$-value & SEM \\
\hline \multicolumn{7}{|l|}{14 days } \\
\hline CAT $(U / m L)$ & $43.90 \pm 2.46$ & $57.21 \pm 13.66$ & $76.80 \pm 44.15$ & $61.52 \pm 31.03$ & 0.445 & 13.960 \\
\hline GSH-Px (U/L) & $90.80 \pm 14.36$ & $115.32 \pm 32.20$ & $145.76 \pm 79.19$ & $120.37 \pm 54.47$ & 0.450 & 23.989 \\
\hline $\mathrm{SOD}(\mathrm{U} / \mathrm{mL})$ & $276.62 \pm 44.10$ & $313.42 \pm 87.16$ & $321.99 \pm 91.99$ & $339.02 \pm 127.71$ & 0.757 & 43.954 \\
\hline $\mathrm{T}-\mathrm{AOC}(\mathrm{U} / \mathrm{mL})$ & $12.25 \pm 2.13$ & $14.33 \pm 4.66$ & $15.40 \pm 4.63$ & $14.79 \pm 6.31$ & 0.753 & 2.211 \\
\hline MDA (nmol/mL) & $6.93 \pm 0.52$ & $8.58 \pm 2.50$ & $8.71 \pm 2.62$ & $8.63 \pm 3.52$ & 0.734 & 1.276 \\
\hline \multicolumn{7}{|l|}{28 days } \\
\hline CAT $(U / m L)$ & $47.93 \pm 2.41 a$ & $45.58 \pm 8.17 a$ & $86.63 \pm 17.73 a b$ & $124.58 \pm 66.85 b$ & 0.020 & 17.563 \\
\hline GSH-Px (U/L) & $90.07 \pm 20.18 \mathrm{a}$ & $108.12 \pm 31.99 a$ & $159.86 \pm 36.77 a b$ & $239.78 \pm 142.58 b$ & 0.043 & 35.992 \\
\hline $\mathrm{SOD}(\mathrm{U} / \mathrm{mL})$ & $230.98 \pm 29.88 a$ & $321.95 \pm 125.37 a$ & $473.54 \pm 125.13 a b$ & $722.33 \pm 418.70 b$ & 0.030 & 108.041 \\
\hline $\mathrm{T}-\mathrm{AOC}(\mathrm{U} / \mathrm{mL})$ & $10.78 \pm 1.62 \mathrm{a}$ & $12.81 \pm 2.82 \mathrm{a}$ & $20.29 \pm 4.68 b$ & $22.72 \pm 7.16 b$ & 0.006 & 2.226 \\
\hline MDA (nmol/mL) & $14.79 \pm 4.97 c$ & $8.43 \pm 3.28 a b$ & $12.47 \pm 2.69 b c$ & $6.70 \pm 1.04 a$ & 0.011 & 1.542 \\
\hline
\end{tabular}




\section{IFN- $\gamma$ and TNF- $a$ mRNA expression}

The expression levels of IFN- $\gamma$ and TNF- $\alpha$ mRNA in the liver, spleen, ileum, and cecum were assessed at 14 and 28 days. As shown in Figs. 1 and 2, the addition of fermented Astragalus to the diets respectively increased the mRNA content on day 14 of IFN- $\gamma$ and TNF- $\alpha$ in the ileum by 1.7 -fold $(P<0.01)$ and 3.1 -fold $(P<0.001)$, and the mRNA content on day 14 of IFN- $\gamma$ in the cecum by 2.1 -fold $(P<0.01)$. Interestingly, we found that the highest amount of IFN- $\gamma$ and TNF- $\alpha$ mRNA in the liver, spleen, ileum, and cecum were present in the fermented Astragalus group at 28 days.

\section{Sequencing output}

A total of 48 intestinal content samples were analyzed by $16 \mathrm{~S}$ rRNA gene sequencing and produced a total of $2,006,223$ high-quality sequences with an average of 41,796 reads. After OUT clustering at 97\% sequence identity, a total of 216,116 OTUs were classified into 49,235 phyla, 48,677 classes, 48,634 orders, 40,101 families, 24,072 genera and 4995 species (Fig. 3).

\section{Diversity of intestinal microflora}

The $\alpha$-diversity of ileal and cecal microbiota of four groups on different days are shown in Table 6. For bacteria on day 14, fermented Astragalus treatment reduced the Chao1 and ACE index in the cecum in comparison to the control treatment suggesting that fermented Astragalus decreased the richness of the bacterial communities. On day 28, the fermented Astragalus treatment increased the estimators of diversity (Shannon and Simpson) of the bacterial community in the ileum. PLSDA was performed to evaluate the similarity ( $\beta$-diversity) of microbial community structure among groups (Fig. 4). PLS-DA plot defined groups where the samples from different groups occupied distinct positions.

\section{Composition of intestinal microflora}

A total of 20 phyla were identified within the intestinal microbiota among 48 samples as shown in Fig. 5. There were three major groups of the intestinal microbiota, including Firmicutes, Bacteroidetes, and Proteobacteria. The relative abundance (\%) of cecal bacterial phyla of hens fed with different dietary supplements was presented in approximately the same amount on days 14 and 28 . On day 28, fermented Astragalus led to a reduced abundance of ileal Firmicutes, with an increased abundance of ileal Bacteroidetes and Proteobacteria. Genus level analysis showed that the Lactobacillus and Bacteroides accounted for the largest proportion of the intestinal microbiota
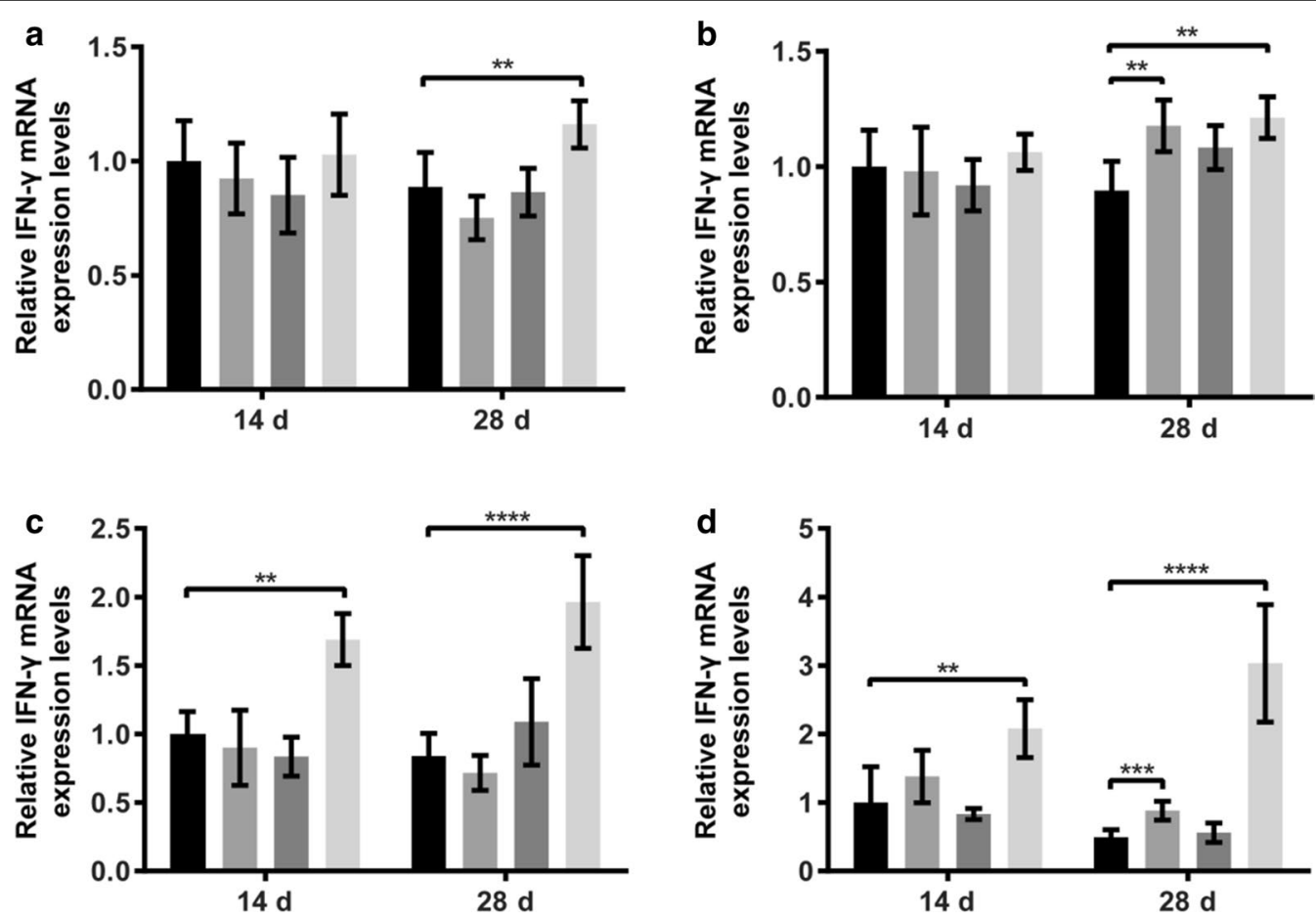

Fig. 1 Effects of different dietary supplements on the relative mRNA expression of IFN- $\gamma$ of laying hens. a Expression levels of IFN- $\gamma$ mRNA in the liver at different time points; $\mathbf{b}$ Expression levels of IFN- $\gamma$ mRNA in the spleen at different time points; $\mathbf{c}$ Expression levels of IFN- $\gamma$ mRNA in the ileum at different time points; $\mathbf{d}$ Expression levels of IFN- $\gamma$ mRNA in the cecum at different time points 

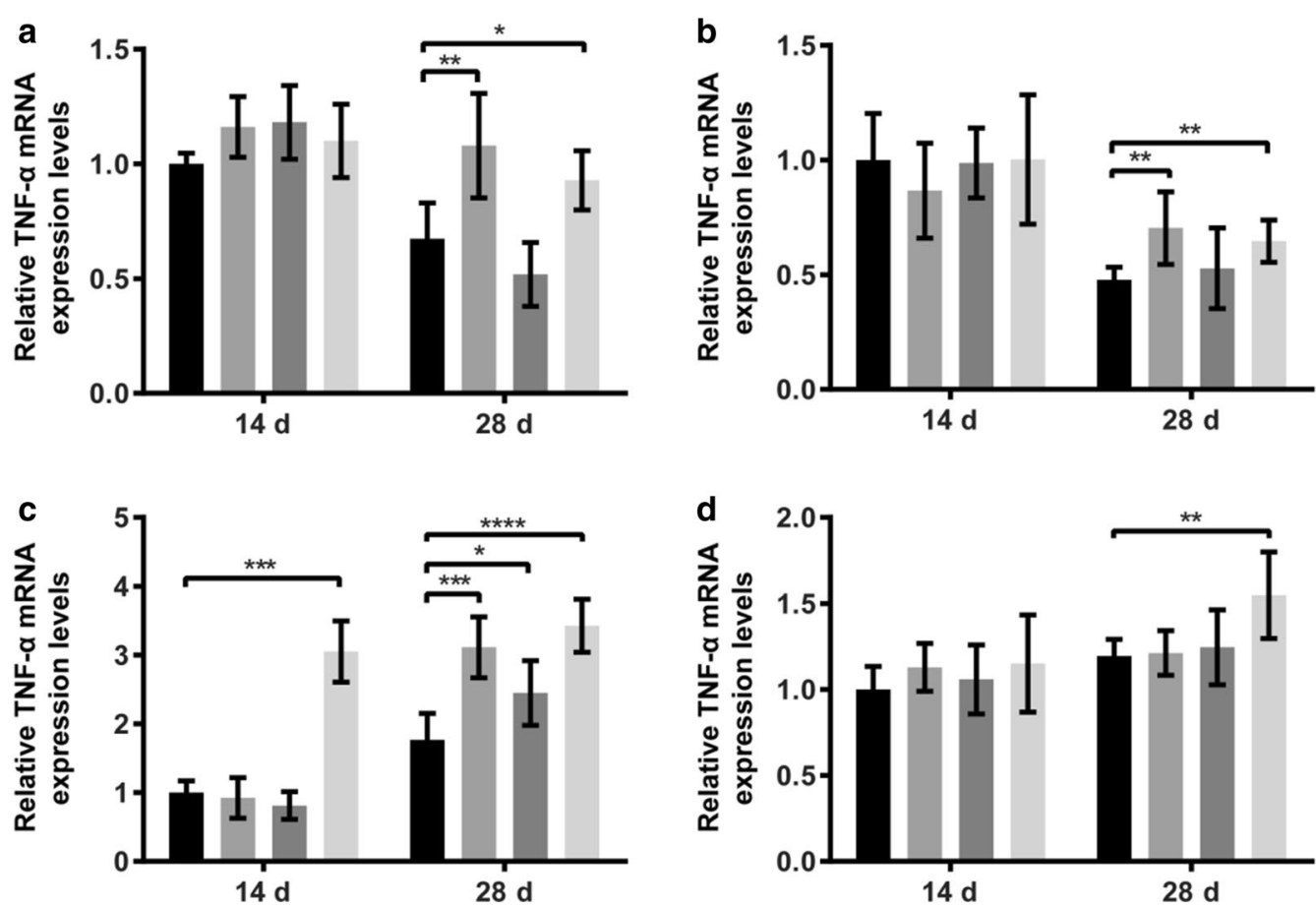

Fig. 2 Effects of different dietary supplements on the relative mRNA expression of TNF-a of laying hens. a Expression levels of TNF-a mRNA in the liver at different time points; $\mathbf{b}$ Expression levels of TNF-a mRNA in the spleen at different time points; $\mathbf{c}$ Expression levels of TNF-a mRNA in the ileum at different time points; $\mathbf{d}$ Expression levels of TNF-a mRNA in the cecum at different time points

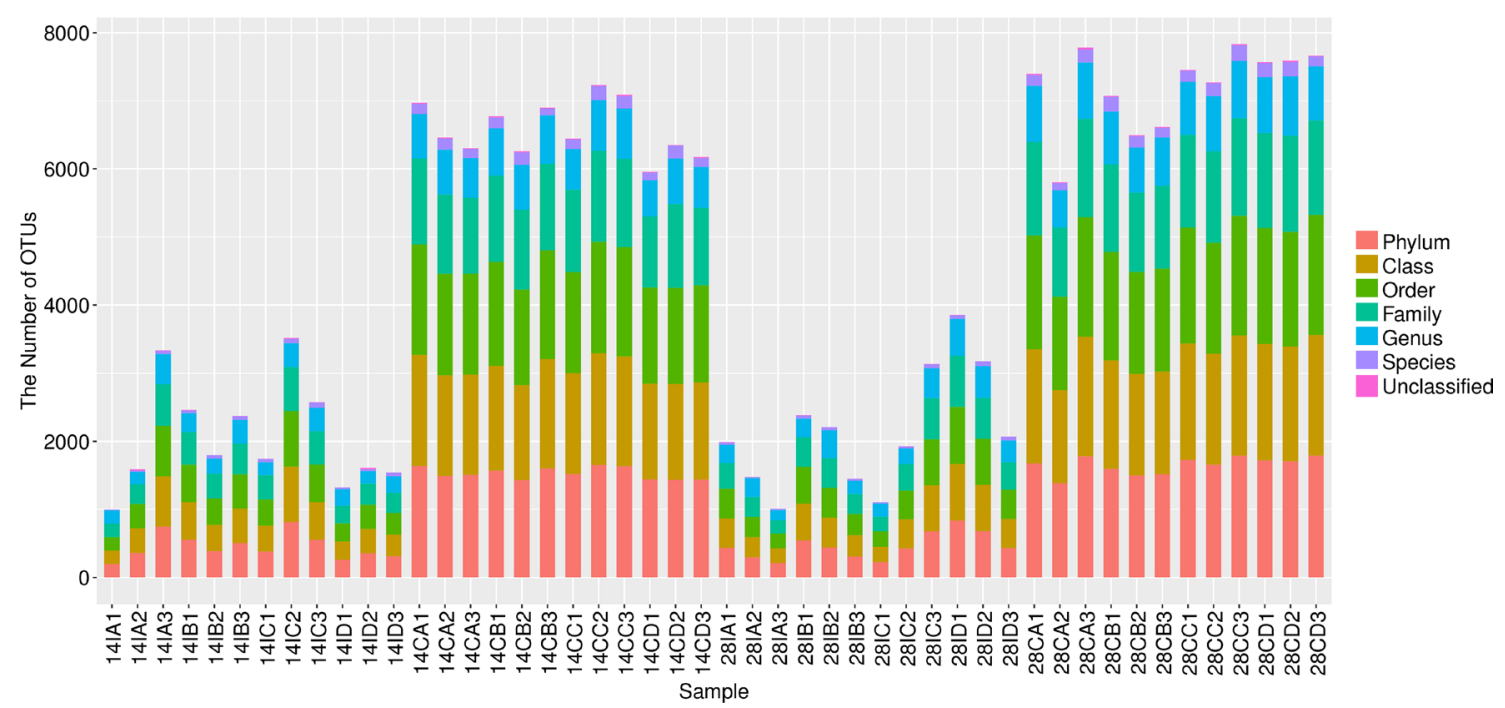

Fig. 3 Number of identified taxa (from phyla to species) among the diverse groups

as shown in Fig. 6. Lactobacillus showed high abundance in the ileum and the extremely low abundance in the cecum. In contrast, Bacteroides showed high abundance in the cecum and the extremely low abundance in the ileum. On day 14, fermented Astragalus addition increased the abundance of cecal Bacteroides by $5.06 \%$ as compared with the control, with no significant influence on the abundance of ileal Lactobacillus. On day 28, 
Table 6 a-diversity indices of ileum and cecum on days 14 and 28

\begin{tabular}{|c|c|c|c|c|c|c|}
\hline Parameters & Control & Astragalus & L. plantarum & Fermented Astragalus & $P$-value & SEM \\
\hline \multicolumn{7}{|l|}{ Ileum-14d } \\
\hline Simpson & $0.84 \pm 0.04$ & $0.84 \pm 0.09$ & $0.84 \pm 0.09$ & $0.79 \pm 0.03$ & 0.846 & 0.045 \\
\hline Chao1 & $476.76 \pm 305.12$ & $526.18 \pm 98.32$ & $649.59 \pm 233.44$ & $337.49 \pm 63.52$ & 0.348 & 115.944 \\
\hline ACE & $493.86 \pm 315.21$ & $550.52 \pm 106.83$ & $673.67 \pm 234.88$ & $346.57 \pm 60.90$ & 0.343 & 118.901 \\
\hline Shannon & $3.88 \pm 2.14$ & $4.73 \pm 0.85$ & $5.28 \pm 1.35$ & $3.29 \pm 1.17$ & 0.402 & 0.844 \\
\hline \multicolumn{7}{|l|}{ Ileum-28d } \\
\hline Simpson & $0.60 \pm 0.01 a$ & $0.55 \pm 0.01 a$ & $0.90 \pm 0.03 b$ & $0.91 \pm 0.04 b$ & 0.000045 & 0.018 \\
\hline Chao1 & $349.78 \pm 139.33$ & $475.76 \pm 144.98$ & $495.18 \pm 269.86$ & $722.42 \pm 210.22$ & 0.219 & 114.547 \\
\hline ACE & $368.44 \pm 146.86$ & $497.43 \pm 146.45$ & $527.56 \pm 288.28$ & $757.36 \pm 217.08$ & 0.222 & 120.155 \\
\hline Shannon & $2.75 \pm 1.10 \mathrm{a}$ & $3.69 \pm 0.89 a b$ & $4.10 \pm 2.10 \mathrm{ab}$ & $5.79 \pm 0.75 b$ & 0.110 & 0.764 \\
\hline \multicolumn{7}{|l|}{ Cecum-14d } \\
\hline Simpson & $0.986 \pm 0.006$ & $0.989 \pm 0.004$ & $0.990 \pm 0.002$ & $0.987 \pm 0.003$ & 0.654 & 0.002 \\
\hline Chao1 & $1816.94 \pm 155.87 b$ & $1944.22 \pm 132.43 b$ & $1923.38 \pm 37.30 \mathrm{~b}$ & $1535.10 \pm 99.29 a$ & 0.009 & 66.509 \\
\hline ACE & $1885.14 \pm 164.82 b$ & $1926.84 \pm 140.76 b$ & $1993.92 \pm 88.19 b$ & $1591.31 \pm 143.86 a$ & 0.030 & 79.294 \\
\hline Shannon & $8.54 \pm 0.15$ & $8.58 \pm 0.30$ & $8.67 \pm 0.12$ & $8.52 \pm 0.24$ & 0.833 & 0.124 \\
\hline \multicolumn{7}{|l|}{ Cecum-28 d } \\
\hline Simpson & $0.99 \pm 0.002 b$ & $0.98 \pm 0.002 \mathrm{a}$ & $0.99 \pm 0.002 b$ & $0.99 \pm 0.003 b$ & 0.026 & 0.001 \\
\hline Chaol & $2008.60 \pm 116.80$ & $1910.78 \pm 63.71$ & $2008.11 \pm 104.08$ & $2022.12 \pm 49.39$ & 0.401 & 47.96 \\
\hline ACE & $2158.80 \pm 107.42 b$ & $1945.56 \pm 76.37 a$ & $2168.85 \pm 123.64 b$ & $2168.34 \pm 34.51 b$ & 0.048 & 51.71 \\
\hline Shannon & $8.73 \pm 0.25 \mathrm{ab}$ & $8.43 \pm 0.17 a$ & $8.73 \pm 0.11 \mathrm{ab}$ & $8.84 \pm 0.18 b$ & 0.109 & 0.106 \\
\hline
\end{tabular}

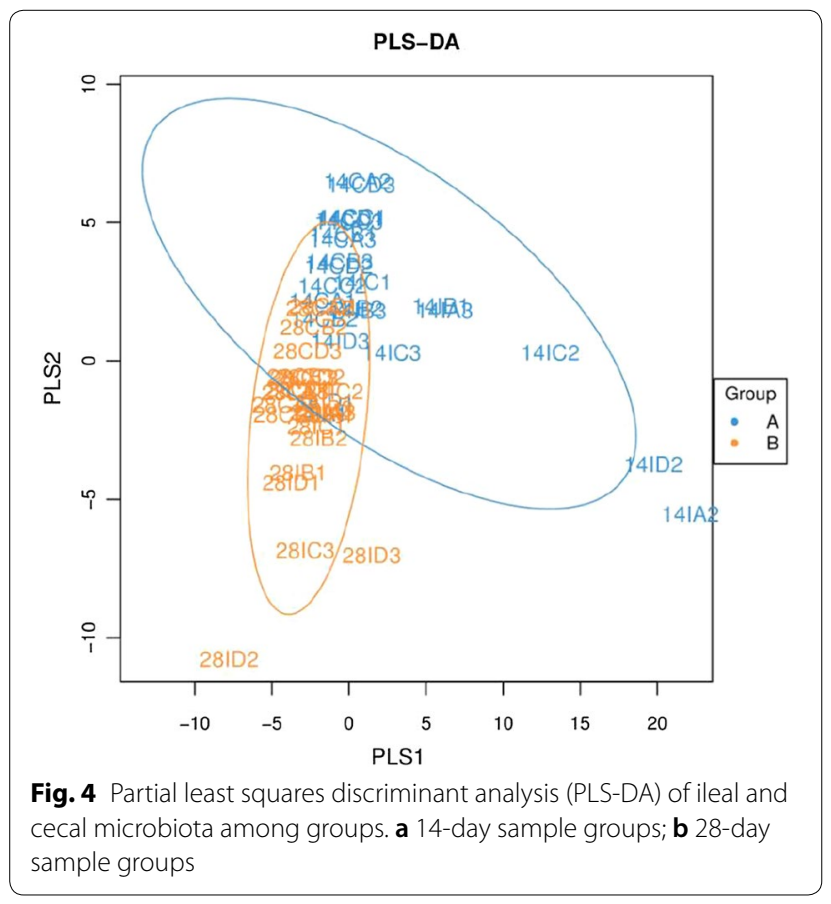

fermented Astragalus addition significantly decreased the abundance of ileal Lactobacillus by $48.51 \%$ as compared with the control, with no significant influence on the abundance of cecal Bacteroides.

\section{Discussion}

The present study was undertaken to investigate the effects of $L$. plantarum fermented Astragalus supplementation on the performance, egg quality, antioxidant status of serum, and gut microbiota in laying hens. When taking out the feeding trial, we observed that diets supplemented with fermented Astragalus increased egg production rate $(P<0.05)$ and decreased feed conversion rate $(P<0.05)$, which may likely be attributed to the improvement of laying hen health status. In vivo, free radicals are harmful by-products generated during normal cellular metabolism and are prone to attack unsaturated fatty acid on the biological membrane, triggering lipid oxidation and lipid peroxide accumulation that result in impairment of organism health (Fang et al. 2002). The antioxidant enzymes CAT, GSH-Px, and SOD are associated with free radical scavenging to protect cells from oxidative damage (Zhang et al. 2014). In the present study, supplementation with fermented Astragalus resulted in the highest levels of CAT, GSH-Px, SOD, and T-AOC and the lowest level of MDA in the serum $(P<0.05)$ on day 28. Our findings are consistent with our previous studies on broilers (Qiao et al. 2018a), indicating that Astragalus fermented by $L$. plantarum can enhance the antioxidant ability of both broilers and laying hens. In our previous 


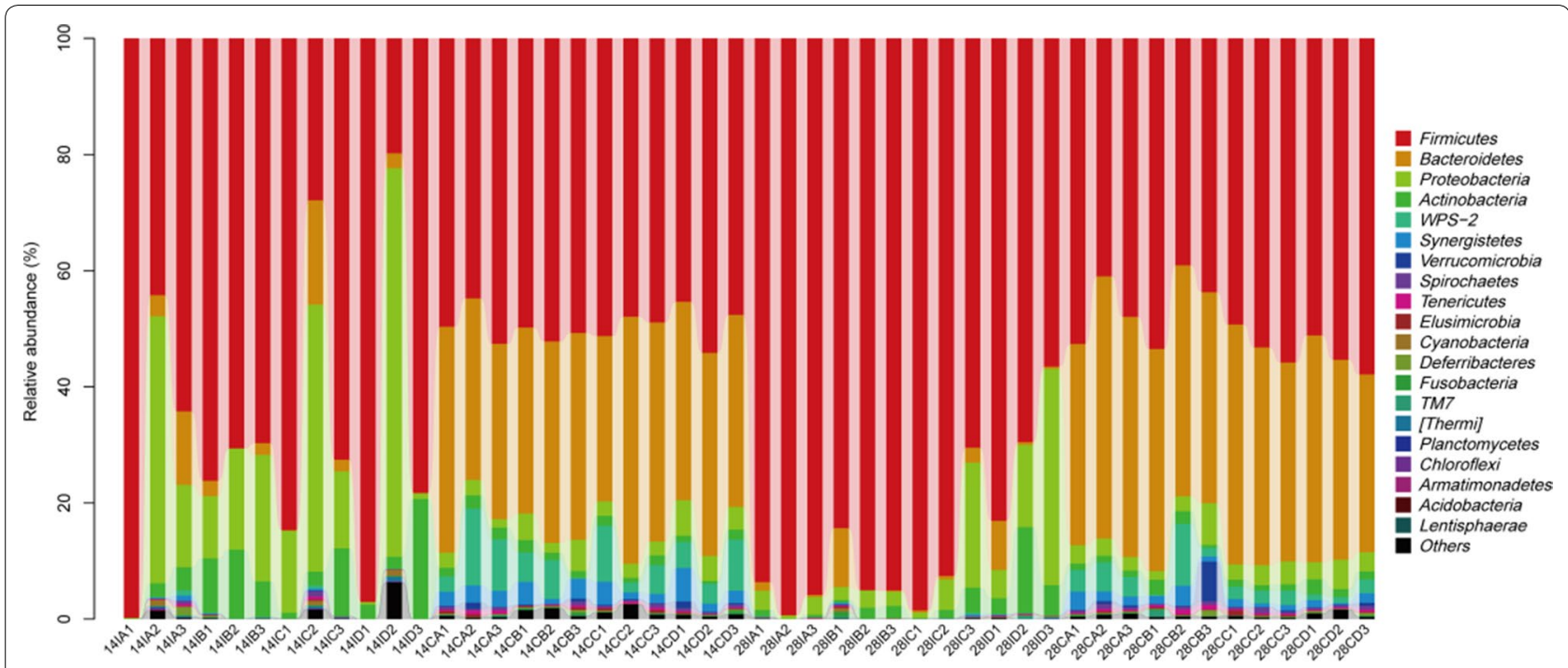

Fig. 5 The phylum level distribution of ileal and cecal microbiota among groups. 14IA: 14-d ileum control group; 14IB: 14-d ileum Astragalus group; 14IC: 14-d ileum L. plantarum group; 14ID: 14-d ileum fermented Astragalus group; 14CA: 14-d cecum control group; 14CB: 14-d cecum Astragalus group; 14CC: 14-d cecum L. plantarum group; 14CD: 14-d cecum fermented Astragalus group; 28IA: 28-d ileum control group; 28IB: 28-d ileum Astragalus group; 28IC: 28-d ileum L. plantarum group; 28ID: 28-d ileum fermented Astragalus group; 28CA: 28-d cecum control group; 28CB: 28-d cecum Astragalus group; 28CC: 28-d cecum L. plantarum group; 28CD: 28-d cecum fermented Astragalus group

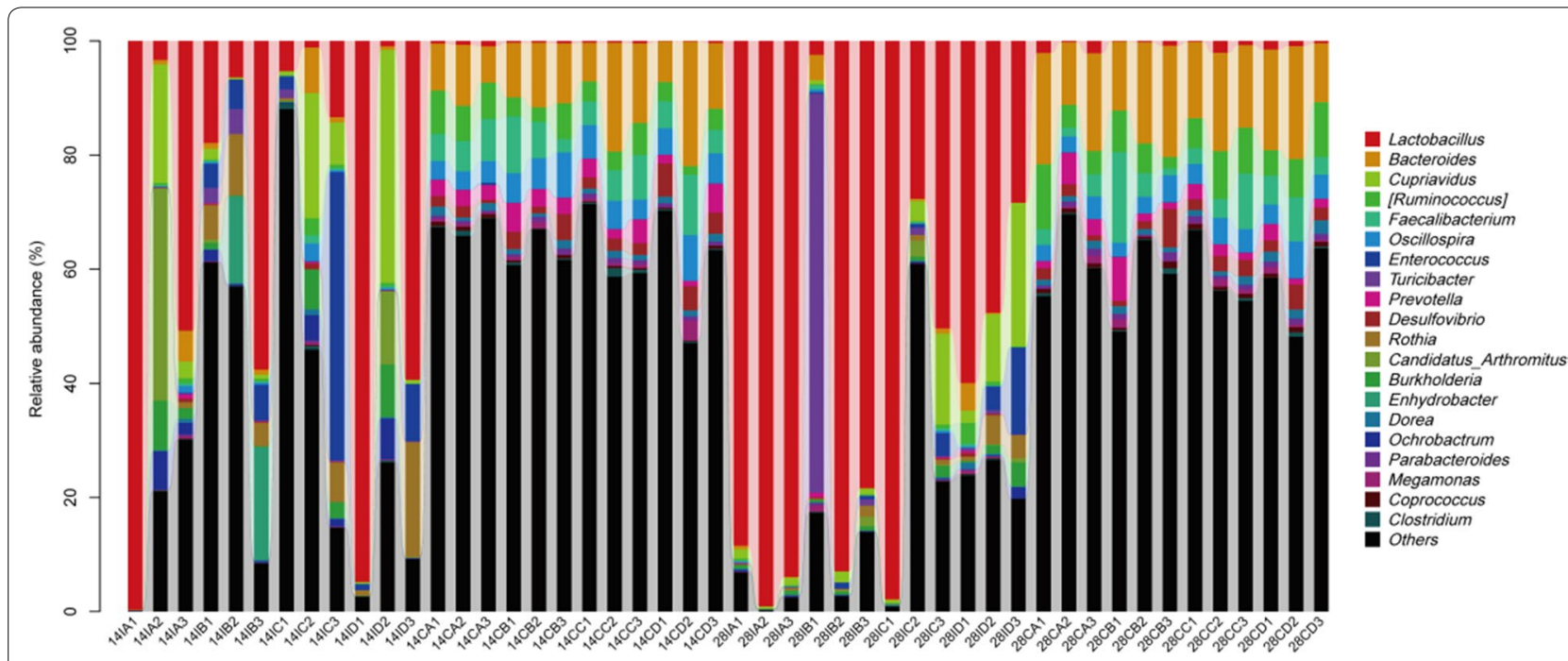

Fig. 6 The genus level distribution of ileal and cecal microbiota among groups. 14IA: 14-d ileum control group; 14IB: 14-d ileum Astragalus group; 14IC: 14-d ileum L. plantarum group; 14ID: 14-d ileum fermented Astragalus group; 14CA: 14-d cecum control group; 14CB: 14-d cecum Astragalus group; 14CC: 14-d cecum L. plantarum group; 14CD: 14-d cecum fermented Astragalus group; 28IA: 28-d ileum control group; 28IB: 28-d ileum Astragalus group; 28IC: 28-d ileum L. plantarum group; 28ID: 28-d ileum fermented Astragalus group; 28CA: 28-d cecum control group; 28CB: 28-d cecum Astragalus group; 28CC: 28-d cecum L. plantarum group; 28CD: 28-d cecum fermented Astragalus group

research, we compared the differences between fermented Astragalus and Astragalus. During the fermentation, the $\mathrm{pH}$ was markedly reduced, as a consequence of the increase of organic acids content, which could inhibit the growth of miscellaneous bacteria (Qiao et al. 2018c). Fermentation could also elevate the content of Astragalus polysaccharide, total saponins, and total flavonoids in Astragalus, and fermented Astragalus possess more abundant microflora (Qiao et al. 2018c). The increase in the content of Astragalus active components by fermentation may be responsible for the enhancement of the performance, egg quality, and antioxidant status of serum 
in laying hens fed with the basal diet supplemented with 3\%o fermented Astragalus.

In recent, Astragalus polysaccharide has attracted rising interests in its anti-cancer effects. A previous study has observed that Astragalus polysaccharide can significantly enhance the proliferation of spleen lymphocytes and increase phagocytosis of peritoneal macrophages in mice and is capable of up-regulating the expression of IL-2, TNF- $\alpha$, and IFN- $\gamma$ in peripheral blood ( $\mathrm{Li}$ et al. 2020). IFN- $\gamma$ and TNF- $\alpha$ are cytokines possessing antitumor and immunomodulatory properties and are essential for host immune responses against infection or tissue injury (Li et al. 2019). At the end of our feeding trial (on day 28), L. plantarum merely increased the mRNA expression of ileal TNF- $\alpha$, Astragalus increased the mRNA expression of splenic and cecal IFN- $\gamma$ and that of hepatic, splenic and cecal TNF- $\alpha$. Interestingly, fermented Astragalus significantly increased the mRNA expression of both IFN- $\gamma$ and TNF- $\alpha$ in all the liver, spleen, ileum, and cecum. However, there are comparatively few findings to date regarding the impact of L. plantarum fermented Astragalus on host immune responses. We speculate that the increased content of Astragalus polysaccharide in the Astragalus after fermentation leads to enhance the body's immune function by increasing the expression of cytokines. Certainly, further investigations will be obliged to fully illustrate that whether there are any endophytic bacteria of Astragalus also responsible for immune activation.

Intestinal microbiota plays a major role in maintaining host health, immunity, and production performance, it has become a research hotspot in recent years (Yeoman et al. 2012). In this study, we also evaluated the effect of fermented Astragalus on intestinal microbiota of laying hens. Our results showed that fermented Astragalus addition increases the diversity of the ileal bacterial community with the increase of feeding time. Furthermore, at the phylum level, Firmicutes, Bacteroidetes, and Proteobacteria were the most dominant phyla in the intestinal microbiota of hens, which are consistent with previous studies (Danzeisen et al. 2011; Yeoman et al. 2012). Interestingly, fermented Astragalus addition led to a reduced abundance of ileal Firmicutes, with an increased abundance of ileal Bacteroidetes and Proteobacteria. We speculate that the increased diversity of the ileal bacterial community might be explained by the fact that the abundance of ileal Firmicutes was reduced to enhance the abundance of other phyla. At the genus level, Lactobacillus as the largest proportion of ileal microbiota of hens is generally highly relevant to feed digestibility (Yan et al. 2017). However, fermented Astragalus addition significantly decreased the abundance of ileal Lactobacillus by $48.51 \%$ as compared with the control at 28 days. These results were totally different from our previous report on the effect of fermented Astragalus on the broiler chicken fecal microbiota, which found that the count of Lactobacillus was increased in chickens fed fermented Astragalus as compared with those in the control group. Those factors responsible for the differences should be further studied.

In conclusion, this study suggested that L. plantarum fermented Astragalus as an efficient dietary additive could significantly promote the production performance, antioxidant capacity, and ileal microbiota diversity of laying hens during the late laying period. A higher expression level of IFN- $\gamma$ and TNF- $\alpha$ in the liver, spleen, ileum, and cecum of laying hens supplemented with fermented Astragalus indicates a particular role of fermented Astragalus on the innate immune system, and this needs a comprehensive investigation in the future to fully illustrate the exact mechanism.

\section{Abbreviations \\ L. plantarum: Lactobacillus plantarum; CAT: Catalase; GSH-Px: Glutathione peroxidase; SOD: Superoxide dismutase; T-AOC: Total antioxidant capacity; MDA: Malondialdehyde; CFU: Colony forming unit; FCR: Feed conversion ratio; ESI: Egg shape index; ESS: Eggshell strength; EST: Eggshell thickness; AH: Albu- men height; HU: Haugh unit; YC: Yolk color; YW: Yolk weight; IFN-Y: Interferon gamma; TNF-a: Tumor necrosis factor-alpha; OTUs: Operational Taxonomic Units; PLS-DA: Partial least squares discriminant analysis.}

\section{Acknowledgements}

Not applicable.

\section{Authors' contributions}

HXQ and HTS contributed to conception and design of the study. HTS, BYW and CZB performed the experiments. HXQ and BYW performed the statistical analysis. $\mathrm{YQH}$ and $\mathrm{HXQ}$ wrote the manuscript. All authors contributed to manuscript revision. All authors read and approved the final manuscript.

\section{Funding}

This study was supported by the Key Discipline of Preventive Veterinary Medicine of Henan University of Animal Husbandry and Economy (mxk2016102), the Research and Innovation Team of Henan University of Animal Husbandry and Economy (2018KYTD13) and the Henan Science and Technology Research Project (192102110076).

\section{Availability of data and materials}

The datasets generated during this study have been deposited in the National Center for Biotechnology Information sequence read archive (https://WwW. ncbi.nlm.nih.gov/biosample) under the Accession number SRA: PRJNA533918.

\section{Ethics approval and consent to participate}

The animal experiments were conducted in accordance with the Guidelines for the Care and Use of Experimental Animals established and approved by the Laboratory Animal Management Committee of Henan University of Animal Husbandry and Economy.

Consent for publication

Not applicable.

Competing interests

The authors declare that they have no competing interests. 


\begin{abstract}
Author details
${ }^{1}$ College of Veterinary Medicine, Henan University of Animal Husbandry and Economy, Zhengzhou 450046, Henan, China. ${ }^{2}$ Key Lab of Freshwater Animal Breeding, Key Laboratory of Agricultural Animal Genetics, Breeding and Reproduction, Ministry of Education, College of Fishery, Huazhong Agricultural University, Wuhan 430070, Hubei, China. ${ }^{3}$ College of Animal Sciences and Veterinary Medicine, Henan Agricultural University, Zhengzhou 450046, Henan, China.
\end{abstract}

Received: 2 June 2020 Accepted: 17 August 2020

Published online: 31 August 2020

\section{References}

Ai S, Tang W, Guo RL, Li JQ, Yang W, He ZG (2019) Research progress on Chinese herbal medicine fermentation and profile of active substances derived. Zhongguo Zhong Yao Za Zhi 44:1110-1118

Bokulich NA, Subramanian S, Faith JJ, Gevers D, Gordon Jl, Knight R, Mills DA, Caporaso JG (2013) Quality-filtering vastly improves diversity estimates from Illumina amplicon sequencing. Nat Methods 10:57-59

Caporaso JG, Kuczynski J, Stombaugh J, Bittinger K, Bushman FD, Costello EK, Fierer N, Peña AG, Goodrich JK, Gordon Jl, Huttley GA, Kelley ST, Knights D, Koenig JE, Ley RE, Lozupone CA, McDonald D, Muegge BD, Pirrung M, Reeder J, Sevinsky JR, Turnbaugh PJ, Walters WA, Widmann J, Yatsunenko T, Zaneveld J, Knight R (2010) QIIME allows analysis of high-throughput community sequencing data. Nat Methods 7:335-336

Danzeisen JL, Kim HB, Isaacson RE, Tu ZJ, Johnson TJ (2011) Modulations of the chicken cecal microbiome and metagenome in response to anticoccidial and growth promoter treatment. PLoS ONE 6:e27949

Fang YZ, Yang S, Wu G (2002) Free radicals, antioxidants, and nutrition. Nutrition 18:872-879

Kallon S, Li X, Ji J, Chen C, Xi Q, Chang S, Xue C, Ma J, Xie Q, Zhang Y (2013) Astragalus polysaccharide enhances immunity and inhibits H9N2 avian influenza virus in vitro and in vivo. J Anim Sci Biotechnol 4:22

Kim JH, Kim MH, Yang G, Huh Y, Kim SH, Yang WM (2013) Effects of topical application of Astragalus membranaceus on allergic dermatitis. Immunopharmacol Immunotoxicol 35:151-156

Li X, Qu L, Dong Y, Han L, Liu E, Fang S, Zhang Y, Wang T (2014) A review of recent research progress on the astragalus genus. Molecules 19:18850-18880

Li Q, Liu C, Yue R, El-Ashram S, Wang J, He X, Zhao D, Zhou X, Xu L (2019) cGAS/ STING/TBK1/IRF3 signaling pathway activates BMDCs maturation following Mycobacterium bovis infection. Int J Mol Sci 20:895

Li W, Hu X, Wang S, Jiao Z, Sun T, Liu T, Song K (2020) Characterization and antitumor bioactivity of astragalus polysaccharides by immunomodulation. Int J Biol Macromol 145:985-997

Pan T, Liu T, Tan S, Wan N, Zhang Y, Li S (2018) Lower selenoprotein T expression and immune response in the immune organs of broilers with exudative diathesis due to selenium deficiency. Biol Trace Elem Res 182:364-372

Patra JK, Das G, Baek KH (2015) Chemical composition and antioxidant and antibacterial activities of an essential oil extracted from an edible seaweed, Laminaria japonica L. Molecules 20:12093-12113
Qiao H, Song Y, Shi H, Bian C (2018a) Fermented Astragalus in diet altered the composition of fecal microbiota in broiler chickens. AMB Express 8:151

Qiao H, Zhang L, Shi H, Song Y, Bian C (2018b) Astragalus affects fecal microbial composition of young hens as determined by $16 \mathrm{~S}$ rRNA sequencing. AMB Express 8:70

Qiao H, Zhang X, Shi H, Song Y, Bian C, Guo A (2018c) Assessment of the physicochemical properties and bacterial composition of Lactobacillus plantarum and Enterococcus faecium-fermented Astragalus membranaceus using single molecule, real-time sequencing technology. Sci Rep 8:11862

Qin Q, Niu J, Wang Z, Xu W, Qiao Z, Gu Y (2012) Astragalus membranaceus inhibits inflammation via phospho-P38 mitogen-activated protein kinase (MAPK) and nuclear factor (NF)-KB pathways in advanced glycation end product-stimulated macrophages. Int J Mol Sci 13:8379-8387

Shahzad M, Shabbir A, Wojcikowski K, Wohlmuth H, Gobe GC (2016) The antioxidant effects of radix Astragali (Astragalus membranaceus and related species) in protecting tissues from injury and disease. Curr Drug Targets 17:1331-1340

Sheih IC, Fang TJ, Wu TK, Chang CH, Chen RY (2011) Purification and properties of a novel phenolic antioxidant from Radix astragali fermented by Aspergillus oryzae M29. J Agric Food Chem 59:6520-6525

Vandemaele F, Vereecken M, Derijcke J, Goddeeris BM (2002) Incidence and antibiotic resistance of pathogenic Escherichia coli among poultry in Belgium. Vet Rec 151:355-356

Wang CZ, Li WJ, Tao R, Ye JZ, Zhang HY (2015) Antiviral activity of a nanoemulsion of polyprenols from ginkgo leaves against influenza A H3N2 and hepatitis B virus in vitro. Molecules 20:5137-5151

Willing BP, Russell SL, Finlay BB (2011) Shifting the balance: antibiotic effects on host-microbiota mutualism. Nat Rev Microbiol 9:233-243

Xu J, Chen HB, Li SL (2017) Understanding the molecular mechanisms of the interplay between herbal medicines and gut microbiota. Med Res Rev 37:1140-1185

Yan W, Sun C, Yuan J, Yang N (2017) Gut metagenomic analysis reveals prominent roles of Lactobacillus and cecal microbiota in chicken feed efficiency. Sci Rep 7:45308

Yeoman CJ, Chia N, Jeraldo P, Sipos M, Goldenfeld ND, White BA (2012) The microbiome of the chicken gastrointestinal tract. Anim Health Res Rev 13:89-99

Zhang Z, Liu D, Yi B, Liao Z, Tang L, Yin D, He M (2014) Taurine supplementation reduces oxidative stress and protects the liver in an iron-overload murine model. Mol Med Rep 10:2255-2262

Zhu H, Cao G, Cai H, Cai B, Hu J (2014) Rapid and undamaged analysis of crude and processed Radix Scrophulariae by Fourier transform infrared spectroscopy coupled with soft independent modeling of class analogy. Pharmacogn Mag 10:265-270

\section{Publisher's Note}

Springer Nature remains neutral with regard to jurisdictional claims in published maps and institutional affiliations.

\section{Submit your manuscript to a SpringerOpen ${ }^{\circ}$ journal and benefit from:}

- Convenient online submission

- Rigorous peer review

- Open access: articles freely available online

- High visibility within the field

Retaining the copyright to your article

Submit your next manuscript at springeropen.com 